\title{
Dedo Azul Não-Isquémico: Caso Raro e Benigno
}

\section{Non-Ischemic Blue Finger: Rare and Benign Case}

Pedro Barreira ${ }^{1}$, André Melicia ${ }^{1}$, João Gagoํㅡㄹ Andreia Fernandes²

Autor Correspondente/Corresponding Author:

Pedro Barreira [pedrobarreira23@gmail.com]

\section{RESUMO}

O dedo azul não-isquémico espontâneo consiste no aparecimento súbito de uma coloração violácea digital isolada, sem outros sintomas associados e de resolução rápida e espontânea. É um fenómeno raro e benigno e constitui um diagnóstico de exclusão, sendo importante realizar o diagnóstico diferencial com outras etiologias mais frequentes, nomeadamente eventos trombóticos, isquémicos ou patologia autoimune. Descreve-se o caso de uma mulher de 83 anos, que recorreu à consulta de uma unidade de saúde por início súbito de cor violácea e edema do terceiro dedo da mão direita, indolor e sem envolvimento da extremidade distal. A avaliação analítica para trombofilias e autoimunidade revelou-se negativa, assim como a investigação imagiológica de isquemia ou trauma. Ao fim de sete dias a doente foi reavaliada, havendo resolução completa do quadro. Este caso assume importância devido à sua semelhança com patologias de prognóstico potencialmente mais grave.

PALAVRAS-CHAVE: Alterações Pigmentação; Dedos; Isquémia

- Autor (es) (ou seu (s) empregador (es)) 2019. Reutilização permitida de acordo com CC BY-NC. Nenhuma reutilização comercial. ${ }^{\circ}$ Author(s) (or their employer(s)) 2019. Re-use permitted under CC BY-NC. No commercial re-use. 


\section{ABSTRACT}

The spontaneous non-ischemic blue finger consists of the sudden onset of isolated digital violaceous discoloration, with no other associated symptoms and rapid and spontaneous resolution. It is a rare and benign phenomenon and represents a diagnosis of exclusion. Thus, it is important to make the differential diagnosis with other more frequent aetiologies, such as thrombotic and ischemic events, or autoimmune pathology. We describe the case of an 83-year-old woman who sought her primary care doctor due to a sudden onset of a painless tumefaction and blueish discoloration of the third finger of her right hand, sparing the distal extremity. The analytical evaluation for thrombophilia and autoimmunity was negative, as was the imaging investigation for ischemia or trauma. The patient was re-evaluated seven days later, showing complete resolution of the condition. This case assumes importance due to its similarity with potentially more serious prognostic pathologies.

KEYWORDS: Fingers; Ischemia; Pigmentation Disorders

\section{INTRODUÇÃO}

O dedo azul não-isquémico espontâneo é uma condição benigna, caracterizada pelo desenvolvimento de uma coloração violácea num dedo, que poupa a extremidade distal, raramente acompanhado de dor. Não afeta outros membros e/ou dedos e tem baixo risco de novos episódios. A etiologia ainda é desconhecida, tendo uma causa diferente da embolização ou do vasoespasmo. A resolução do quadro é rápida e espontânea, sem precisar de qualquer tratamento. ${ }^{1,2}$

É uma entidade rara, que pode ocorrer em todas as faixas etárias, afetando predominantemente a população feminina de meia-idade (idade média de 57 anos). Trata-se de um diagnóstico de exclusão, sendo importante realizar diagnóstico diferencial com outras patologias cujas manifestações clínicas são semelhantes, sejam elas trombóticas, embólicas ou autoimunes.

O fenómeno de Raynaud e a doença vascular periférica são hipóteses frequentes na marcha diagnóstica. O fenómeno de Raynaud distingue-se por poder envolver os dedos das mãos e dos pés, geralmente mais do que um dedo e de forma simétrica em cada episódio, não poupando a extremidade distal. As alterações cutâneas habitualmente apresentam um padrão variável entre palidez, cianose ou hiperemia. No fenómeno de Raynaud primário, a mediana da idade de apresentação é de 14 anos, com início raramente após 30 anos. Já no caso do fenómeno de Raynaud secundário, a população afetada encontra-se na faixa de idade superior a 30 anos e os doentes apresentam características clínicas sugestivas da doença de base, principalmente doenças do tecido conjuntivo (e.g.: alteração nas provas de função respiratórias, artrite). O dedo azul não-isquémico não parece ser desencadeado pelo frio ou stress, nem surge associado a patologia autoimune reumatológica. ${ }^{3-5}$
Relativamente à doença vascular periférica, esta tende a afetar uma população mais idosa que a do dedo azul não-isquémico, com predomínio no sexo masculino. Doentes com trombose digital espontânea apresentam, desde início, coloração menos exuberante e nódulos ao exame físico. Em comparação com fenómenos isquémicos, estes adquirem uma coloração mais roxa e denota-se ausência dos pulsos periféricos, bem como diminuição da temperatura. Em todos relatos de dedo azul não-isquémico não houve menção de fenómenos

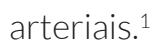

Se nenhuma doença sistémica for identificada, muito provavelmente a condição resolve-se espontaneamente, sendo rara ocorrência de novos episódios.

\section{CASO CLÍNICO}

Mulher, 83 anos, recorre a consulta aguda no Centro de Saúde acompanhada pelo filho. Vem por aparecimento súbito de cor violácea e edema do terceiro dedo da mão direita, com evolução de um dia. Negava trauma, dor ou alteração da sensibilidade. Referiu episódio anterior há vários anos com resolução espontânea. Não apresentava outras queixas associadas.

Apresenta antecedentes pessoais de hipertensão arterial controlada e doença refluxo gastroesofágico. Mantinha como medicação habitual perindopril + indapamida 5/1,25 mg, lercanidipina $10 \mathrm{mg}$ e esomeprazol $20 \mathrm{mg}$.

Ao exame objetivo apresentava cor violácea no terceiro dedo da mão direita (Fig.s 1 e 2), mas que poupava a extremidade distal, com edema ligeiro, sem comprometimento funcional. Os pulsos radial e cubital estavam mantidos e simétricos. Não tinha alteração na sensibilidade ou da mobilidade. Indolor à palpação. Pele com temperatura normal. Apirética (temperatura axilar: 


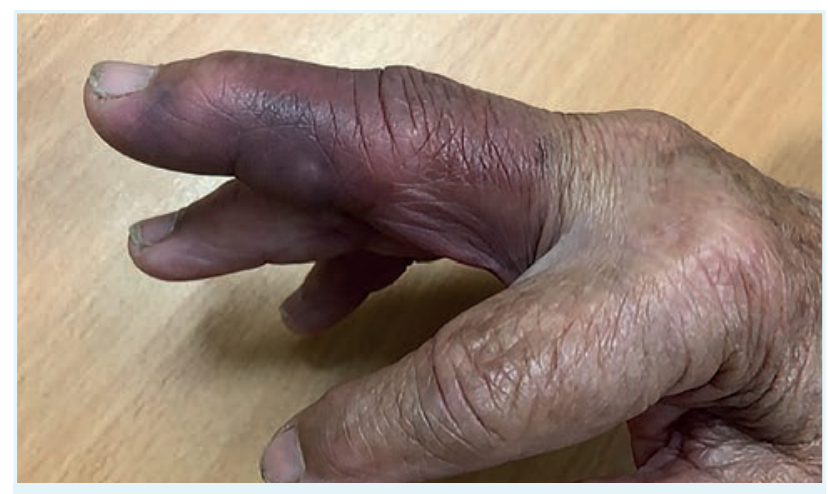

FIGURA 1. Coloração violácea no dedo da mão direita (lateral).

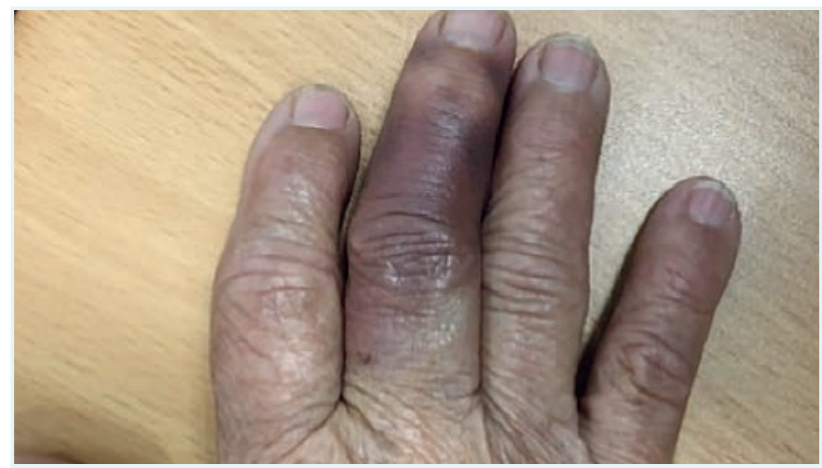

FIGURA 2. Coloração violácea no dedo da mão direita (dorsal).

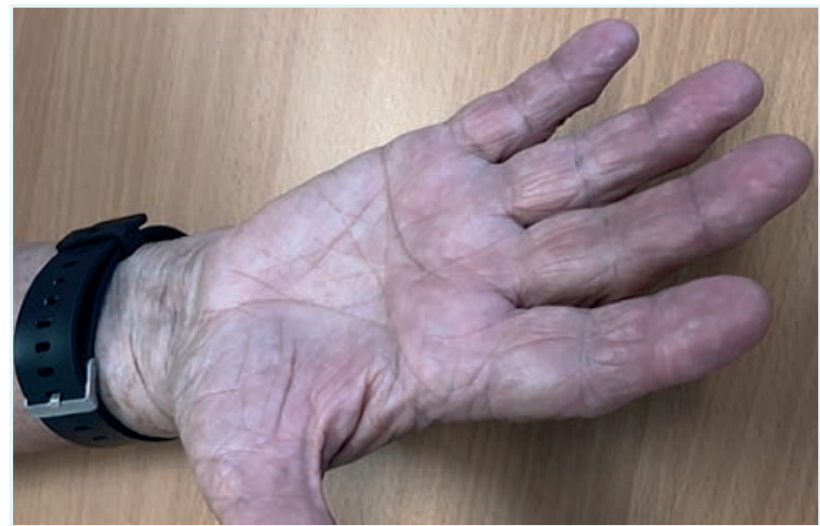

FIGURA 3. Coloração normal do dedo da mão direita (vista palmar).

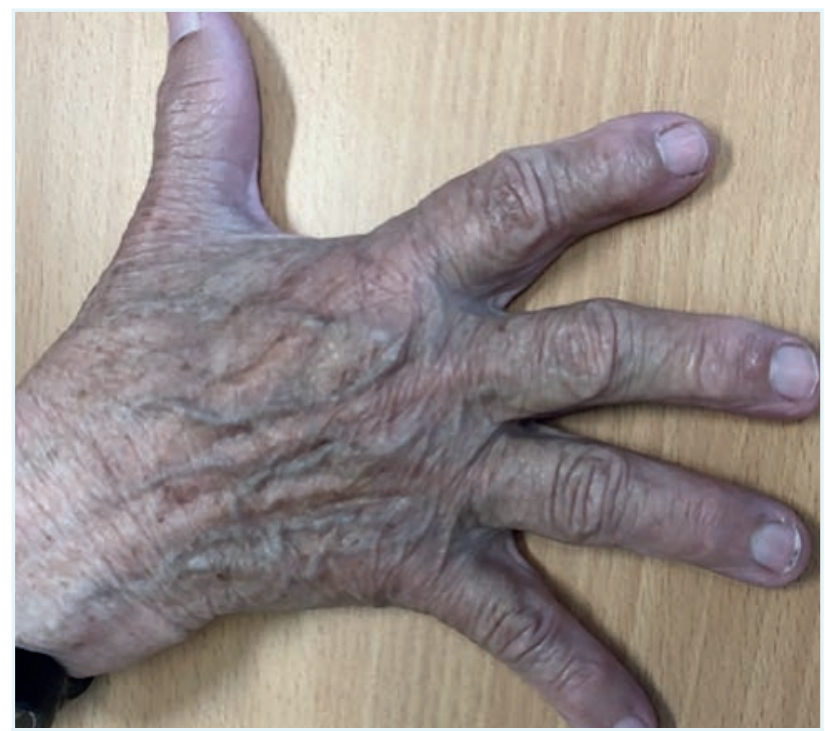

FIGURA 4. Coloração normal do dedo da mão direita (vista dorsal). $37,0^{\circ} \mathrm{C}$ ), saturação periférica de $\mathrm{O}_{2}$ de $98 \%$ em ar ambiente, normotensa (TA: 134/77 mmHg), com frequência cardíaca de 68 bpm.

À data da consulta foi colocada a hipótese de diagnóstico de dedo azul não-isquémico, tendo sido prescrita a realização em ambulatório de análises para exclusão de trombofilias e doenças autoimunes. Foi também requisitada ecografia partes moles urgente para exclusão de isquemia ou trauma, apesar da sua aparente baixa probabilidade pela história clínica. Explicaram-se os sinais que deveriam motivar a ida ao serviço de urgência, tendo ficado agendada consulta de reavaliação.

A reobservação no Centro de Saúde teve lugar sete dias depois. Ao exame objetivo a doente apresentava resolução completa dos sintomas (Fig.s 3 e 4), com coloração normal do dedo e sem qualquer dor. Referiu que a cor violácea desapareceu de forma espontânea após 72 horas. $O$ estudo analítico para diagnóstico diferencial e a ecografia das partes moles foi negativo (Tabelas 1 e 2).

\section{CONCLUSÃO}

A etiologia do dedo azul não-isquémico ainda é desconhecida, não surgindo associado a fenómenos arteriais. Os episódios de características agudas podem ser precipitados por atividades de vida diária, mas não estão relacionados com extremos térmicos. Não há evidência de doença sistémica associada ou ingestão de drogas. Esta síndrome não tem significado clínico para o doente,

TABELA 1. Avaliação analítica.

\begin{tabular}{|l|c|c|}
\hline Parâmetros & Resultado & Valor referência \\
\hline Hemoglobina & $14,4 \mathrm{~g} / \mathrm{dL}$ & $12-15$ \\
\hline Hematócrito & $44,6 \%$ & $36-46$ \\
\hline Volume globular médio & $93 \mathrm{fL}$ & $80-97$ \\
\hline Leucócitos & $9,0 \times 10^{9} / \mathrm{L}$ & $4-10$ \\
\hline Neutrófilos & $52,1 \%$ & $40-80$ \\
\hline Linfócitos & $37,5 \%$ & $20-40$ \\
\hline Velocidade de sedimentação & $10 \mathrm{~mm} / \mathrm{s}$ & $3-12$ \\
\hline Proteína C reativa & $0,7 \mathrm{mg} / \mathrm{dL}$ & $<1,0$ \\
\hline Anti-trombina funcional normal & $123 \%$ & $75-125$ \\
\hline Proteína C funcional normal & $138 \%$ & $70-140$ \\
\hline Proteína S funcional normal & $113 \%$ & $52-118$ \\
\hline Fator de von Willebrand & $150 \%$ & $66,1-176,3$ \\
\hline Anticorpos anti-CCP & Negativo & $<7$ \\
\hline
\end{tabular}

TABELA 2. Avaliação imagiológica.

\begin{tabular}{|c|c|}
\hline $\begin{array}{l}\text { Ecografia de } \\
\text { partes moles } \\
\text { (mão direita) }\end{array}$ & $\begin{array}{l}\text { Alterações degenerativas das interfalângicas com } \\
\text { maior expressão na interfalângica proximal onde } \\
\text { há pequena quantidade de líquido intra-articular. } \\
\text { Não há alterações das estruturas tendinosas. } \\
\text { Não há alteração do tecido subcutâneo. }\end{array}$ \\
\hline
\end{tabular}


mas é importante que os médicos estejam cientes dela, porque a fase aguda pode causar ansiedade, pela sua semelhança com doença vascular grave.

CONFLITOS DE INTERESSE: Os autores declaram não ter qualquer conflito de interesse na realização do presente trabalho.

FONTES DE FINANCIAMENTO: Não houve qualquer fonte de financiamento na realização do presente trabalho.

CONFIDENCIALIDADE DOS DADOS: Os autores declaram ter seguido os protocolos da sua instituição acerca da publicação dos dados de doentes.

CONSENTIMENTO: Consentimento do doente para publicação obtido.

PROVENIÊNCIA E REVISÃO POR PARES: Não comissionado; revisão externa por pares.

CONFLICTS OF INTEREST: The authors declare that they have no conflicts of interest.

FINANCIAL SUPPORT: This work has not received any contribution, grant or scholarship.

CONFIDENTIALITY OF DATA: The authors declare that they have followed the protocols of their work center on the publication of data from patients.

PATIENT CONSENT: Consent for publication was obtained.

PROVENANCE AND PEER REVIEW: Not commissioned; externally peer reviewed.

\section{REFERÊNCIAS}

1. Batra M, Tandon P, Gupta N. Clinical aproach to a patient with isolated digital ischaemia. JIACM. 2002;3:23-8.

2. Khaira HS, Rittoo D, Vohra RK, Smith SRG. The non-ischaemic blue finger. Ann R Coll Surg Engl. 2001;83:154-7.

3. Weinberg I, Jaff M. Spontaneus blue finger syndrome: a benign process. Am J Med. 2012;125:e1-2. doi: 10.1016/j.amjmed.2011.05.007.

4. Cowen R, Richards T, DharmadasaA, HandaA, Perkins JMT. The acute blue finger: management and outcome. Ann R Coll Surg Engl. 2008; 90:557-60. doi: 10.1308/003588408X318237.

5. Deliss LJ, Wilson JN. Acute blue fingers in women. J Bone Joint Surg Br. 1982;64:458-9. 